\title{
Electronic Structure of Superlattice and Twin in Ga Doped ZnO Measured by Monochromated EELS
}

\author{
H.J. Chang, * S.W. Yoon, *** T.Y. Seong**, T.H. Yu***, Y.H. You***, J.P. Ahn*
}

*Nano Materials Analysis Center, Korea Institute of Science and Technology, Hwarangno 14-gil 5, Seongbuk-gu, Seoul 136-791, Korea

**Department of Materials Science and Engineering, Korea University, Seoul 136-713, Korea

***Electronic Materials Lab, Samsung Corning Precision Materials, 644-1, Jinpyeong-dong, Gumi, 730-735, Korea

Transparent conducting oxide thin films are necessary for use as transparent electrodes in flat panel displays such as liquid crystal displays (LCDs), plasma display panels, electronic paper displays and touch panels. At present, indium-tin-oxide (ITO) thin films prepared by magnetron sputtering deposition methods are in practical use for most thin-film transparent electrode applications. However, the difficulty in supply of In because of the high cost and scarcity of indium caused development of substitute materials for ITO transparent electrodes. It has been noted that the best, and only practical, indium-free candidate for an alternative material to ITO is impurity-doped $\mathrm{ZnO}$ such as Al- or Ga-doped $\mathrm{ZnO}$ (AZO or GZO). Here, we focused on the fundamental effect of the dopants on the microstructure and the electronic structure of the GZO target material. Particularly the reasons of high conductivity through the characterization of plane defects, crystal orientation, doping contents, crystal structure in $\mathrm{Zn}_{1-\mathrm{x}} \mathrm{Ga}_{\mathrm{x}} \mathrm{O}(\mathrm{x}=0,2.7,5.6$, 6.1, 6.6 wt.\%).

We manufactured $\mathrm{Zn}_{1-\mathrm{x}} \mathrm{Ga}_{\mathrm{x}} \mathrm{O}$ targets by sintering $\mathrm{ZnO}$ and $\mathrm{Ga}_{2} \mathrm{O}_{3}$ powders, having a theoretical density of $99.9 \%$ and homogeneous Ga-dopant distribution in $\mathrm{ZnO}$ grains. The microstructure was carried out by using rotation holder (650 single tilt rotation analytical holder, Gatan) in (S)TEMs (FEI Tecnai F20 G ${ }^{2}$ and Titan S 80-300 operated at $200 \mathrm{kV}$ and $300 \mathrm{kV}$, respectively). The electronic structure was investigated by VEELS, using a Gatan imaging filtering system, attached to the Titan at $80 \mathrm{kV}$. The microscope is equipped with a monochromated gun, providing a best energy resolution of $0.16 \mathrm{eV}$.

The effect of doping was studied by measuring the electric conductivity by Van der Pauw method with different concentrations of $\mathrm{Ga}$. Pure $\mathrm{ZnO}$ shows relatively high resistivity (1.26 $\Omega \mathrm{cm}$ ) at room temperature. With the addition of $\mathrm{Ga}$, however, the resistivity decreases by three orders of magnitude representing $3.26 \times 10^{-3} \Omega \mathrm{cm}$ at $5.6 \mathrm{wt} \% \mathrm{Ga}$, beyond which the resistivity was found to increase again. It has been found that $\mathrm{Ga}$ can substitute into $\mathrm{ZnO}$ lattice resulting in an lattice expansion along the c-axis $(2 \sim 5 \%)$ and biaxial compressive stress along the in-plane direction [1]. However, only $0.5 \%$ of lattice expansion was measured by XRD analysis in this study. Instead, defects such as superlattices and twins were observed in TEM images which may relax the stress in the lattices. The correlation between the electric conductivity and defects were found by mapping the distribution of superlattices, twins and grains with no defects over $>100$ grains by TEM. In the sample of $5.6 \mathrm{wt} \%$ GZO which exhibited lowest conductivity shows highest fraction of grains with superlattice $(\sim 73.5 \%)$ compared with the values in neighboring compositions (3.4\% in 27. Wt.\% GZO and $70.9 \%$ in $6.4 \mathrm{wt} \% \mathrm{GZO}$ ). Therefore it can be concluded that superlattice by Ga atoms contribute significantly to the increase of the electric conductivity. 
In order to prove the effect of defects on the electric properties it is necessary to compare the local electrical conductivities of the grains with superlattices or twins. (S)TEM/EELS is powerful tool to characterize the electronic structure in those small grains. First, the raw EEL spectra taken from $5.6 \mathrm{wt} . \% \mathrm{GZO}$ with/without the gun monochromator were compared in Fig. 2. It is noticed that monochromated EEL spectrum exhibits different low loss features as well as the improved energy resolution. Thus the bandgap measurement which requires the accurate spectrum particularly in the low energy region below $5 \mathrm{eV}$ was performed with the monochromated gun and the loss function was normalized by Kramers-Kronig sum rule and the imaginary part of the dielectric function were compared for the superlattices and twins.

\section{References}

[1] C.H. Ahn, et al., Nanotechnology, 20 (2009) 015601.

[2] This research is sponsored by the Pioneer Research Center Program through the National Research Foundation of Korea funded by the Ministry of Education, Science and Technology.
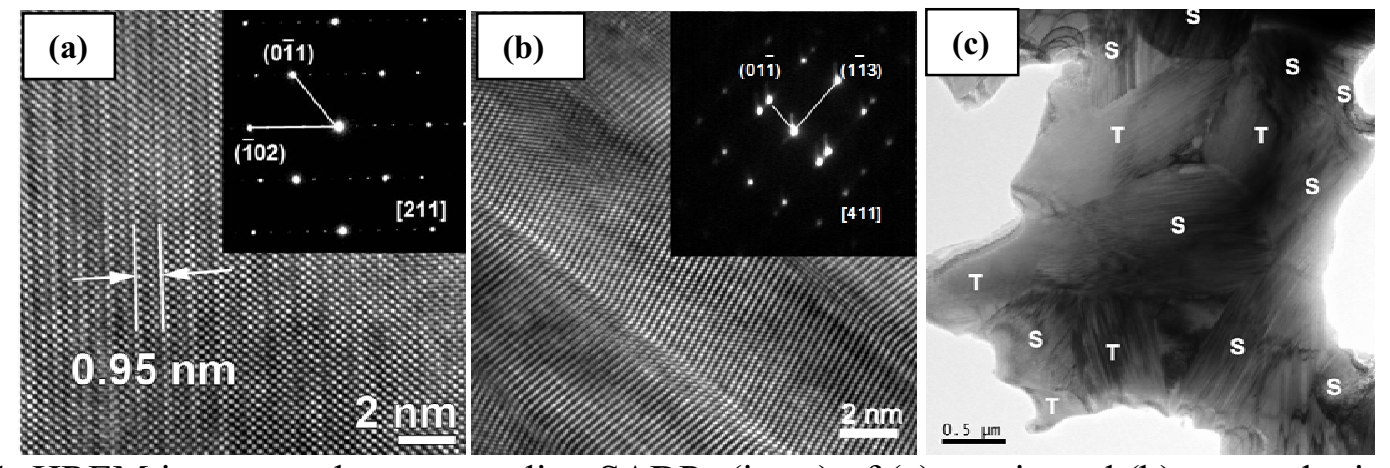

FIG. 1. HREM images and corresponding SADPs (inset) of (a) a twin and (b) a superlattice. The twins observed from [411] zone axis exhibit spacing of $\sim 4 \mathrm{~nm}$. It was found that the ordering ratio of pairs of substitution atoms depends on the zone axis, that is $1 / 3$ from [001], 1/4 from [011], [111], [211], [101] and 1/5 from [211]. The inset SADP of (a) obtained from [211] shows the substitution of one Ga layer out of 5 unitcells. The twin plane in (b) was determined as (1-13) from systematic diffraction patterns analysis.; (c) Bright field image obtained from 5.6wt $\% \mathrm{Ga}$ doped $\mathrm{ZnO}$ showing the distribution of twins $(\mathrm{T})$ and superlattices $(\mathrm{S})$.

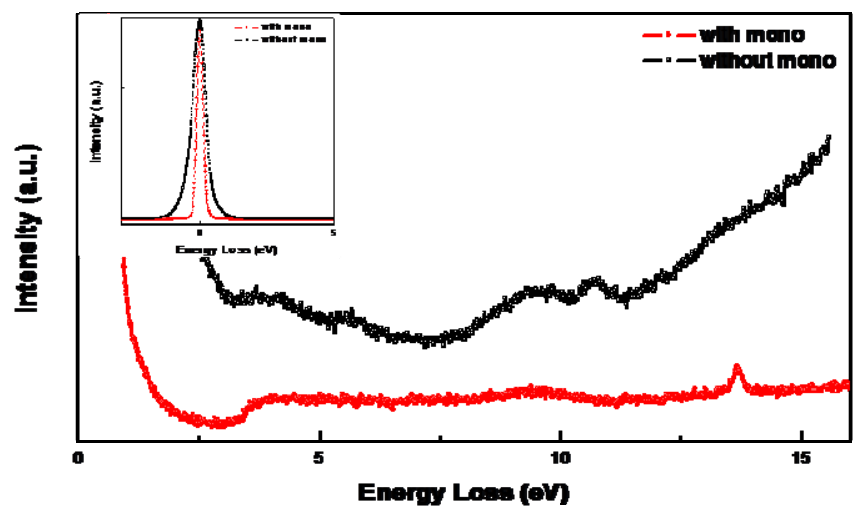

Fig. 2. Comparison of low loss EEL spectra taken from 5.6 wt.\% GZO with / without the monochromated gun (inset) and the enlarged low energy loss regions. 